\title{
Effective Color Image Retrieval Based on the Gaussian Mixture Model
}

\author{
Maria Luszczkiewicz-Piatek ${ }^{1}$ and Bogdan Smolka ${ }^{2, \star}$ \\ 1 University of Lodz, Faculty of Mathematics and Computer Science, \\ Department of Applied Computer Science, Banacha 22, 90-238 Lodz, Poland \\ mluszczkiewicz@math.uni.lodz.pl \\ 2 Silesian University of Technology, Department of Automatic Control, \\ Akademicka 16 Str, 44-100 Gliwice, Poland \\ bogdan.smolka@polsl.pl
}

\begin{abstract}
The main problem addressed in this paper is as follows: a system applying the proposed framework should retrieve all images whose color structure is similar to that of the given query image, independently on the applied lossy coding. We propose an approach based on the color histogram approximation using the Gaussian Mixture Model. The proposed method incorporates the information on the spatial distribution of the color image pixels utilizing the bilateral filtering scheme. The retrieval results were evaluated on large databases of natural color images and the usefulness of the proposed technique was compared with some commonly known retrieval methods operating on color histograms.
\end{abstract}

Keywords: color image retrieval, lossy compression, Gaussian mixture.

\section{Introduction}

Over the recent years, many spectacular technological achievements have revolutionized the way information is acquired and handled. Nowadays, more than ever, there is an exponentially growing number of images being captured, stored and made available on the Internet. However, managing this huge amount of visual information for retrieval purposes, especially when a very specific retrieval criterion is set, still remains a challenging task and therefore there are many attempts to address it [1,2].

Spatial organization of colors has been recently explored in form of spatial statistics between color pixels, such correlograms or some filter responses [3, 4, 5, 6]. Related approaches use points of interest similarly to classic object recognition methods [7] and many other retrieval methods rely on segmentation as a basis for image indexing [8 9 10 11]. Mutual arrangements of regions in images are also the basis of the retrieval, however the representation of the relationship can be non-symbolic [12].

The idea of parametric color image description is used in many image analysis solutions. Although several proposed image retrieval techniques utilize the Gaussian Mixture Model (GMM) [13, 14] as color distribution descriptor [15, 16], the aspect of the distortions caused by the lossy compression was not taken into account. These methods simply index all images in the database by fitting GMM to the data, according to some predefined rules.

\footnotetext{
* Corresponding author.
}

R. Schettini, S. Tominaga, and A. Trémeau (Eds.): CCIW 2011, LNCS 6626, pp. 199 213.2011.

(C) Springer-Verlag Berlin Heidelberg 2011 
The lossy compression significantly corrupts the color distribution of an image and a lack of the application of any refinement techniques may lead to the high rate of false negative results, as images stored in lossy formats are considered as dissimilar on the basis of their corrupted color palette. The solutions proposed in this work aim to to overcome these difficulties through the modifications of the GMM procedure [17, 18, 19, 20, 21]. In details, in the proposed method the GMM is used as a descriptor of the image color distribution. Its advantage is that it overcomes the problems connected with high dimensionality of standard color histograms. However, the very important aspect is the fact that the proposed method, based on weighted two-dimensional Gaussians is robust to distortions introduced by lossy compression techniques and therefore it can be used for the retrieval of images contained in the Web based databases, which very often store images in lossy compression formats, like JPG.

The second group of issues analyzed in this paper is related to the important problem of the construction of a histogram sensitive to the color image composition. The drawback of various kinds of standard color histograms is that they do not take into account the spatial image structure, which is crucial in many applications such as object recognition and image categorization. Moreover, the proposed solution can be useful in retrieving images of the same content but differently arranged within the image as a result of the changes in image scene between capture moments. Therefore, images with the same color proportions, but with different color arrangements, have the same chromaticity histogram. Thus, the goal is to take into account the spatial arrangement of the image pixels. Therefore, we propose to use the weighting coefficients provided by the Bilateral Filter (BF) [22, 23], which considers the chromatic and topological similarity of neighboring image pixels.

The paper is organized as follows. The details of the new technique are described in Section 2 . Section 3 presents the experimental setup of the retrieval, based on the proposed solution. The comparison of the experimental results for various compression schemes is also presented. In this section we also focus on the spatial arrangement of the color pixels within the scene depicted in the image. The comparison with other methods operating on color histograms is presented in Section 4 . Finally, Section 5 concludes the concepts and results presented in this paper.

\section{Gaussian Mixture Modeling}

The first and very important decision concerning the color image data modeling is the choice of the color space suitable for the retrieval experiments [24]. In this paper we are using mainly the normalized rgb space (independent on the color intensity) and the results were also evaluated for the CIE Lab color space [25].

The first step in applying the proposed methodology is to construct the histogram $H(x, y)$ in the rg chromaticity space defined as $H(x, y)=N^{-1} \sharp\left\{r_{i, j}=x, g_{i, j}=y\right\}$, where $H(x, y)$ denotes a specified bin of a two-dimensional histogram with $r$ component equal to $x$ and $g$ component equal to $y$, the symbol $\sharp$ denotes the number of samples in a bin and $N$ is the number of color image pixels.

The next stage of the presented technique is the modeling of the color histogram using the Gaussian Mixture Models (GMM) and utilizing the Expectation-Maximization (EM) algorithm for the model parameters estimation as described in details in [13,26]. 
The input data are realizations of a variable $X$, which are the $(r, g)$ or $(a, b)$ pairs (referring to rgb or CIE Lab) of the color image pixels: $X=\left(x_{1,1}, \ldots, x_{n_{1}, n_{2}}\right)$, where $n_{1}, n_{2}$ denote image sizes. The probability density function $p(X \mid \Theta)$ of color data is governed by a set of parameters $\Theta$. The GMM parameters are the mean and covariance matrix for each Gaussian function. We assume that the data items are independent and identically distributed and the resulting distribution density $p$ of the data samples is $p(X \mid \Theta)=\prod_{i=1}^{N} p\left(x_{i} \mid \Theta\right)=\mathcal{L}(\Theta \mid X)$. The function $\mathcal{L}(\Theta \mid X)$ is a likelihood function or likelihood of the parameters given the data. The goal of the estimation procedure is to find $\Theta^{*}$ which maximizes $\mathcal{L}: \Theta^{*}=\arg \max _{\Theta} \mathcal{L}(\Theta \mid X)$. Usually we maximize $\log \mathcal{L}(\Theta \mid \mathcal{X})$ as it is analytically more convenient.

Let us assume the following probabilistic model:

$$
p(x \mid \Theta)=\sum_{m=1}^{M} \alpha_{m} p_{m}\left(x \mid \theta_{m}\right),
$$

which is composed of $M$ components and its parameters are defined as:

$\Theta=\left(\alpha_{1}, \ldots \alpha_{M}, \theta_{1}, \ldots, \theta_{M}\right)$, with $\sum_{m=1}^{M} \alpha_{m}=1$. Moreover, each $p_{m}$ is a function of the probability density function which is parameterized by $\theta_{m}$. Thus, the analyzed model consists of $M$ components with $M$ weighting coefficients $\alpha_{m}$.

Finally after derivations shown in [26] the model parameters are defined as:

$$
\begin{gathered}
\alpha_{m}^{k+1}=N^{-1} \sum_{i=1}^{N} p\left(m \mid x_{i}, \Theta^{k}\right), \mu_{m}^{k+1}=\frac{\sum_{i=1}^{N} x_{i} \cdot p\left(m \mid x_{i}, \Theta^{k}\right)}{\sum_{i=1}^{N} p\left(m \mid x_{i}, \Theta^{k}\right)} \\
v_{m}^{k+1}=\frac{\sum_{i=1}^{N} p\left(m \mid x_{i}, \Theta^{k}\right)\left(x_{i}-\mu_{m}^{k+1}\right)\left(x_{i}-\mu_{m}^{k+1}\right)^{T}}{\sum_{i=1}^{N} p\left(m \mid x_{i}, \Theta^{k}\right)}
\end{gathered}
$$

where $\mu$ and $v$ denote the mean and variance, $m$ is the index of the model component and $k$ is the iteration number. The $\mathrm{E}$ (Expectation) and $\mathrm{M}$ (Maximization) steps are performed simultaneously, according to (2) and (3) and in each iteration, as the input data we use parameters obtained in the previous one.

The main idea of the application of the GMM technique lies in the highly desirable properties of this approach. The inherent feature of the GMM enables to approximate the distorted color histogram of the color image subjected to lossy compression. The analyzed test image shown in Fig 1 a, was compressed to $15 \%$ of it's file size using JPG method. The lossy compression causes a loss of color information resulting in discontinuities in the chromaticity histogram (Figs. 1k and 1 d ). The proposed methodology counteracts this effect by smoothing the histogram in order to reconstruct its original shape (Figs.11-h), which is a basis for the effective image retrieval.

In the classical approach to the construction of the chromaticity histograms, the spatial arrangement of the color pixels in an image is not considered, and therefore e.g. two images of distinctly different content, but the same color palette, are recognized as very similar and related to the same query. This is illustrated by an example in Fig. 2. Each of the query images is composed of the same number of colors in the same proportions, but these colors are arranged in different spatial structures and each of these images has exactly the same chromaticity histogram. The perceptual difference perceived by 
(a)

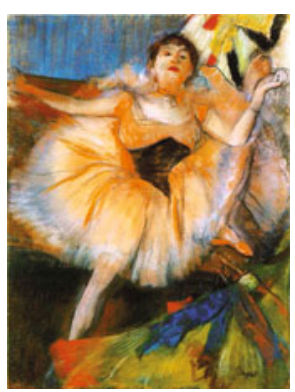

(c)

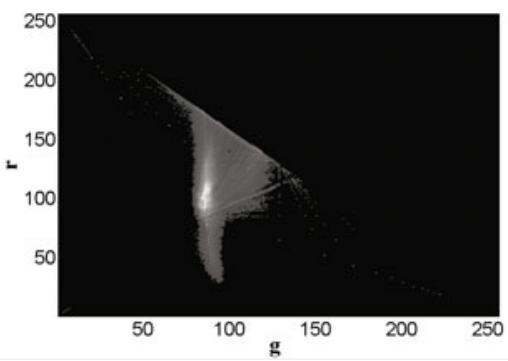

(e)

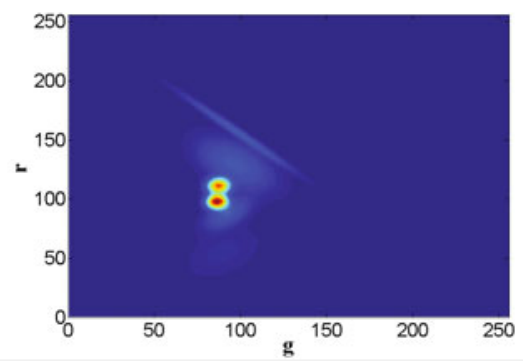

(g)

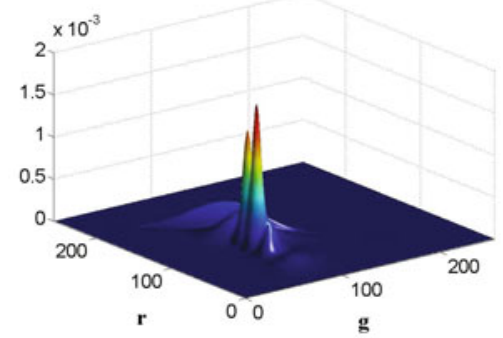

(b)

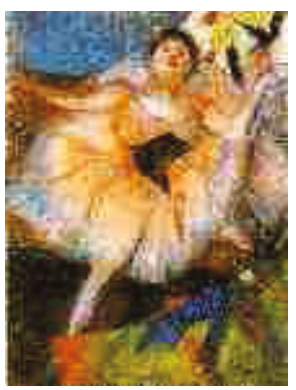

(d)

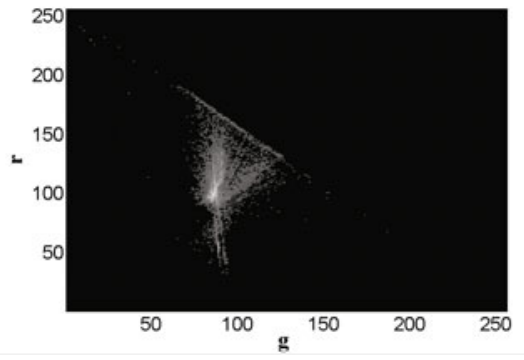

(f)

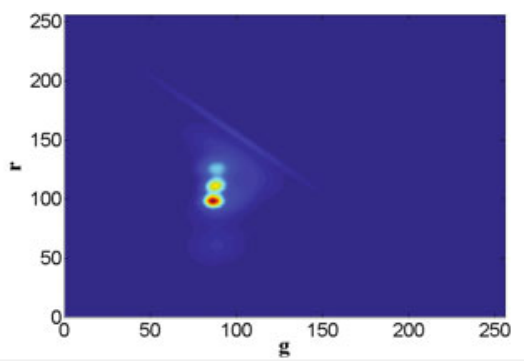

(h)

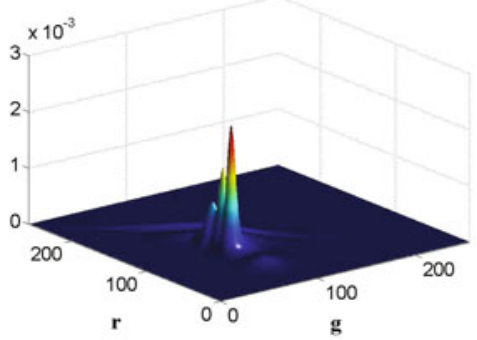

Fig. 1. Comparison of test rg histograms and their GMM models for an original image (a) and its JPG version (b). The second row (c, d) shows the rg histograms and the third row depicts the 2D visualizations of GMM of original image (e) and its JPG version (f). The bottom row presents the $3 \mathrm{D}$ visualizations of the GMM for analyzed images $(\mathrm{g}, \mathrm{h})$. 


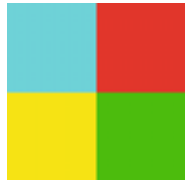

$I_{1}$

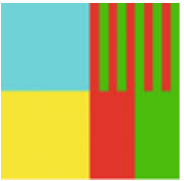

$I_{2}$

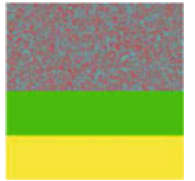

$I_{3}$

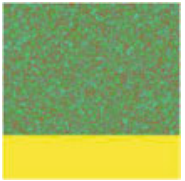

Color histograms

of queries $I_{1}-I_{4}$

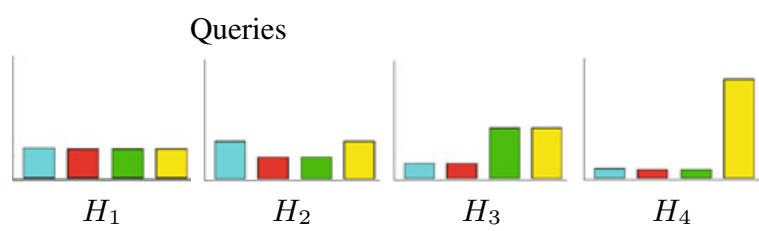

Desired chromaticity histograms

Fig. 2. The color histogram evaluated without taking into account the spatial arrangement of the colors contained in the query image does not allow to discriminate between images of various color arrangements (upper row). The desired histogram construction should reflect the spatial arrangement of the colors contained in the images (lower row).

an observer is not reflected by their common color histogram. To describe these differences, the procedure of color histogram construction must be modified to report more accurately the differences between these images.

Thus, in order to reflect the differences in image content and also to improve the retrieval efficiency of the proposed retrieval scheme the concept of Bilateral Filtering (BF) [22, 23], was incorporated. Its use provides a possibility to emphasize in the rg color histogram all the pixels belonging to homogenous color areas. In general, its construction reflects two factors related with topological closeness and color similarity of image pixels.

Using the concept of BF, each pixel of the original image is taken into the rg histogram with the weight evaluated on the basis of the closeness to its neighbors and their color similarity. Thus, chromaticity histogram is in fact a histogram of weights associated to color pixels. Each bin value is the sum of the weights of the pixels of the particular $r-g$ or $a-b$ values.

In details, the weights assigned to the pixel at position $(x, y)$ are computed according to the following scheme:

$$
w_{x, y}=\frac{1}{n} \sum_{(i, j) \in W} \exp \left(-\frac{\left\|\boldsymbol{c}_{x, y}-\boldsymbol{c}_{i, j}\right\|}{h}\right)^{k_{1}} \cdot \exp \left(-\frac{d_{i, j}}{\delta}\right)^{k_{2}},
$$

where $\boldsymbol{c}_{i, j}$ and $\boldsymbol{c}_{x, y}$ denote the color pixels at positions $(i, j)$ and $(x, y)$ respectively, $h$ is the color difference scaling parameter, $d_{i, j}$ is the Euclidean distance between the pixel at position $(i, j)$ and $(x, y)$, which is the center of the filtering window $W$ and $\delta$ is a spatial normalizing parameter equal to the diameter of the square filtering window. The number of pixels $n$ in $W$ was set to be equal to $10 \%$ of the total number of pixels in the image and we assumed $k_{1}=k_{2}$. 


\subsection{Initial Conditions of the GMM}

conditions on the basis of the clustering algorithms such as the k-means method [14]. Other approaches specify the starting values and randomly divide data to $M$ classes and assign them to $M$ groups corresponding to $M$ model components. In practice, it is equivalent to the randomly generated set of integers from the range $(1, M)$ corresponding to each of the observation $x_{i}$.

The proposed variation of a random start, suitable for the presented methodology, comprises the random generation of independent mean values $\mu_{i}^{(0)}$ derived from normal distribution, denoted as $G: \mu_{1}^{(0)}, \ldots, \mu_{m}^{(0)}, G(\bar{x}, \Sigma)$, where $\bar{x}$ is the mean of the analyzed samples of the data and $\Sigma$ is the sample covariance matrix.

For the purposes of the histogram comparisons, several distance measures were used (denoted as $d$ ) and computed between the histogram of the evaluated image (denoted as $\mathrm{H}$ ) and the 2D surface generated by the GMM model of its histogram, (denoted as C):

$$
\begin{aligned}
& \text { - } \mathrm{L}_{1} \mathrm{D}: \quad d(H, C)=\sum_{i=1}^{\eta} \sum_{j=1}^{\eta}\left|H_{i, j}-C_{i, j}\right|, \\
& \text { - } \mathrm{L}_{2} \mathrm{D}: \quad d(H, C)=\left(\sum_{i=1}^{\eta} \sum_{j=1}^{\eta}\left|H_{i, j}-C_{i, j}\right|^{2}\right)^{\frac{1}{2}}, \\
& \text { - Bhattacharyya Distance, }(\mathrm{BD}): \quad d(H, C)=\sum_{i=1}^{\eta} \sum_{j=1}^{\eta} \sqrt{H_{i, j} \cdot C_{i, j}}
\end{aligned}
$$

Extensive experiments lead to the conclusion that 7 components are sufficient to approximate the structure of a color histogram of lossy compressed images, and thus this number of components is adequate to reconstruct the original histogram structure with no prior knowledge about its construction. Figure 3 a illustrates the exemplary modeling of lossy compressed images. It can be noticed that the increase of model complexity does not result in significant fitness improvement of the data derived from the model to uncorrupted data.

Additional experiments were conducted in order to estimate the number of necessary iterations of the EM algorithm. For each histogram structure 31 experiments were evaluated, for 7 model components, setting various starting conditions of the algorithm. The coefficients $\alpha$ were assigned randomly and drawn from uniform probability density function.

The obtained results (see Fig. 3b) lead to the conclusion that small number of iterations, such as 75, (in some cases even 50) are fairly enough to reflect the histogram structure independently on the used compression scheme. The further increase of the number of iterations will not significantly ameliorate the fitness of the data derived from the model to original color information.

For validating the accuracy of the modeling, the original test images were subjected to various compression techniques, such as JPG and JPG2000, and modeled using the GMM of 7 components with 75 iterations of the EM algorithm. For each of the images, fitting was done for 31 random initial values. The evaluated results in the form of the box plots are shown in Fig 3 r. It can be noticed that the obtained results are in general comparable for all tested compression schemes. 


\section{Experimental Setup for GMM Based Image Retrieval}

The experiments were performed in the following manner. The GMM parameters associated with all of the images in the chosen database were computed and saved in advance, so that only a similarity matrix is used for retrieving the most similar answers to the given query among all the candidate images in the database. The estimation of the GMM was done for 7 components with 75 iterations.

Having models parameters computed, a comparison between the histogram of the query image and of those belonging to the image database was performed and the images were ordered according to the values of the previously described $\mathrm{L}_{1} \mathrm{D}, \mathrm{L}_{2} \mathrm{D}$ and $\mathrm{BD}$ distance measures. Due to the fact that $\mathrm{L}_{2} \mathrm{D}$ produced irrelevant retrieval results it was excluded from the further experiments.

For the evaluation of the difference between two histograms we also used the the Earth Mover's Distance (EMD) similarity measure. The EMD is based on the assumption that one of the histograms reflects "hills" and the second represents "holes" in the ground of a histogram. The measured distance is defined as a minimum amount of work needed to transform one histogram into the other using a "soil" of the first histogram. As this method operates on signatures and their weights using GMM, we assigned as signature values the mean of each component and for the signature weight the weighting coefficient of each Gaussian in the model. The EMD computations were evaluated using the Y. Rubner algorithm implementation [27].

As can be seen in Fig. 4 the proposed method enables efficient retrieval of lossy compressed images. The results evaluated for images represented by their rg histogram, illustrate that the proposed method can successfully counteract the loss of color information. The suitability of the proposed method is especially visible when, for demonstration purposes, the GIF compression scheme, which severely distorts the color information was applied.

As it was mentioned before, the best solution to the problems addressed in this paper should be based on the perceptual similarity of colors which are related to their distance in a given space. Moreover, the color similarity measure should preferably offer compact color description. Therefore, the CIE Lab color space combined with the Earth Mover's distance promises to fulfil such a requirement.

Figure 5 illustrates the comparison between retrieval results evaluated for exemplary images of the Wang database using GMM approximation of color histograms evaluated in CIE Lab and rgb color spaces. Those results were compared to a set of candidate images produced by the retrieval system based only on color histograms which were not subjected to GMM modeling mixture (last rows in Fig. 5). The results show that the described methodology overcomes the drawbacks of the method based on histograms derived for images represented in rgb color space. Additionally, the application of the CIE Lab color space enables to fully utilize the qualities of the EMD measure providing accurate results even for highly compressed color images.

For the illustration of the proposed method, the Webmuseum and our database was chosen. Figure 6 shows the retrieval results evaluated for various settings of the BF parameters. The choice of the best set is rather a subjective matter as each of the shown 


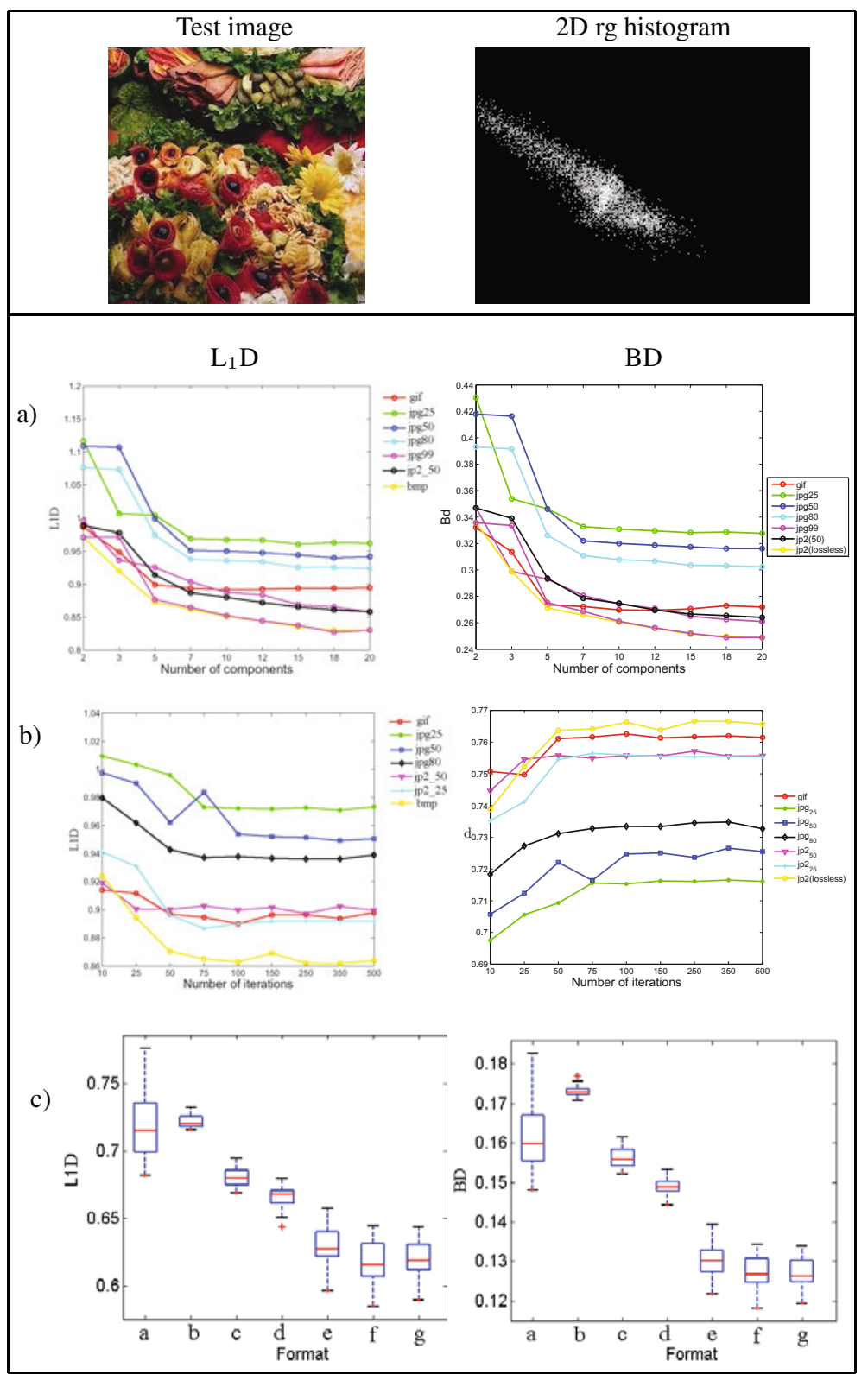

Fig. 3. Comparison between the histogram of the test image and its approximations obtained through the GMM using $\mathrm{L}_{1} \mathrm{D}$ and $\mathrm{BD}$ distances for various number of model components (a) and iterations (b) of the estimation process performed by the EM algorithm. The bottom row (c) illustrates the statistical description of the modeling when using 7 components and 75 iterations of EM for various compression formats. 
sets of candidate images is satisfactory. This example also illustrates the ability of the proposed technique to not only retrieve images of the same overall color palette, but also to promote images with homogenous color regions.

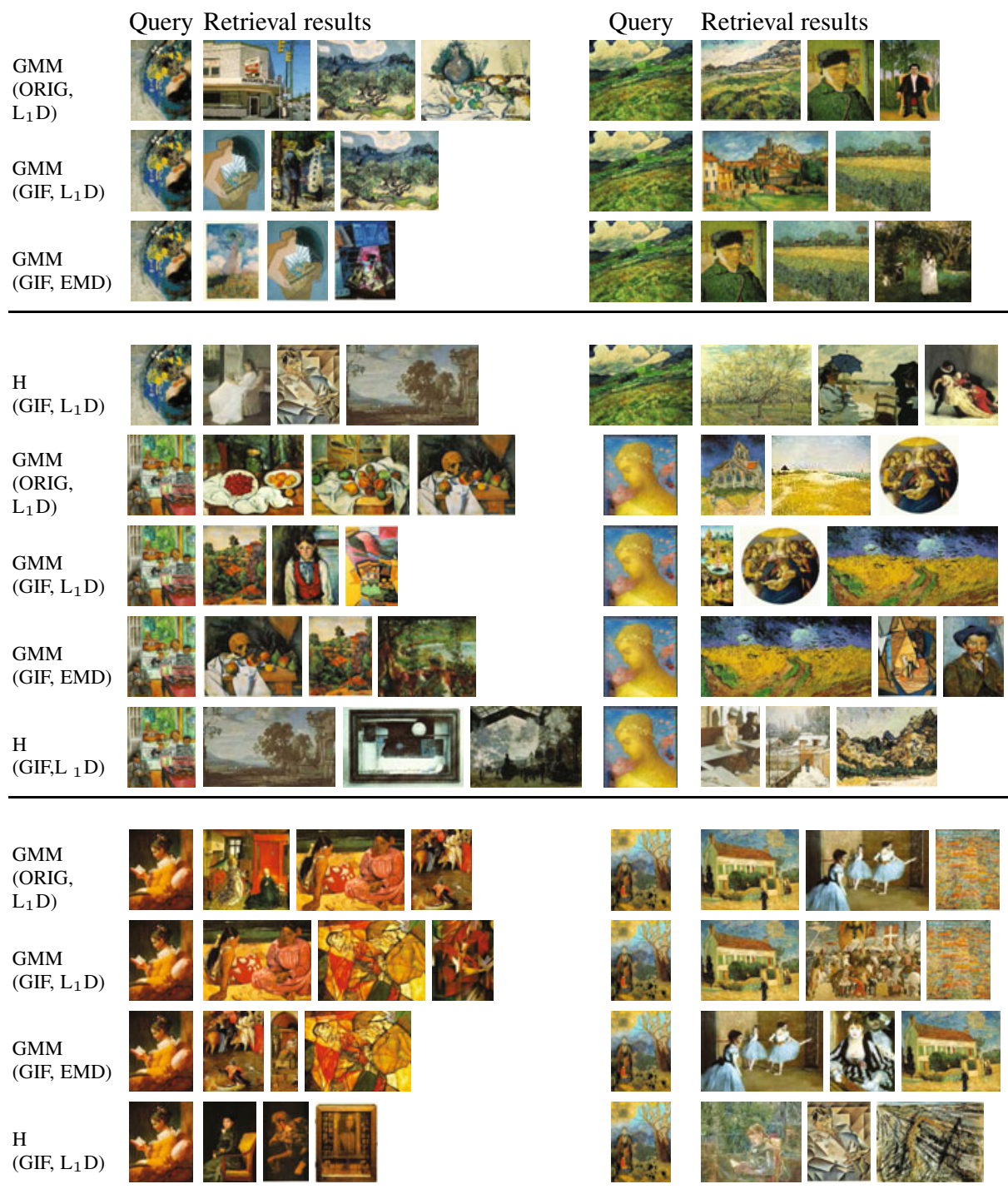

Fig. 4. Retrieval results for exemplary original images (denoted as ORIG) chosen from the database of Webmuseum and its color palette decreased versions. The GIF technique was applied as it facilitates the illustration of the changes caused by color information loss. Retrieval process was evaluated according to $\mathrm{L}_{1}$ metric as a distance measure in rgb color space. For each query (left) three highest ranked images are shown. 


\section{GIF}

$(\mathrm{EMD}, \mathrm{rgb})$
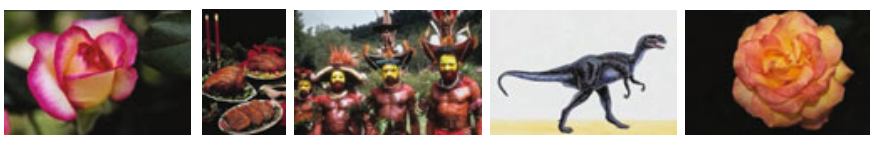

GIF
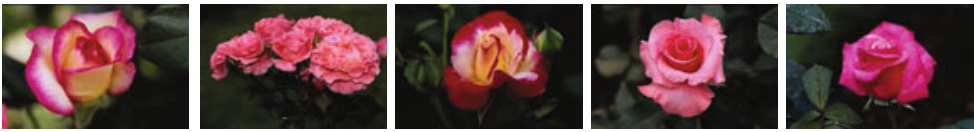

(EMD, Lab)
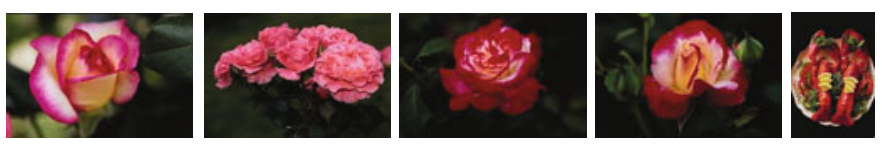

(EMD, Lab)
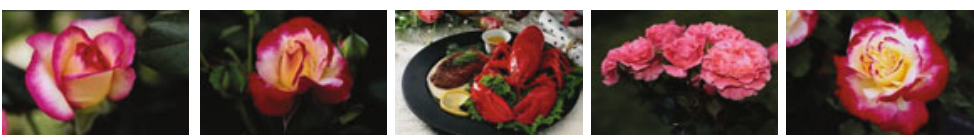

(EMD, Lab)

$\mathrm{H}$
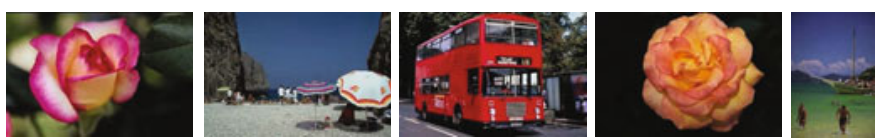

(GIF, $\left.\mathrm{L}_{1} \mathrm{D}, \mathrm{rgb}\right)$

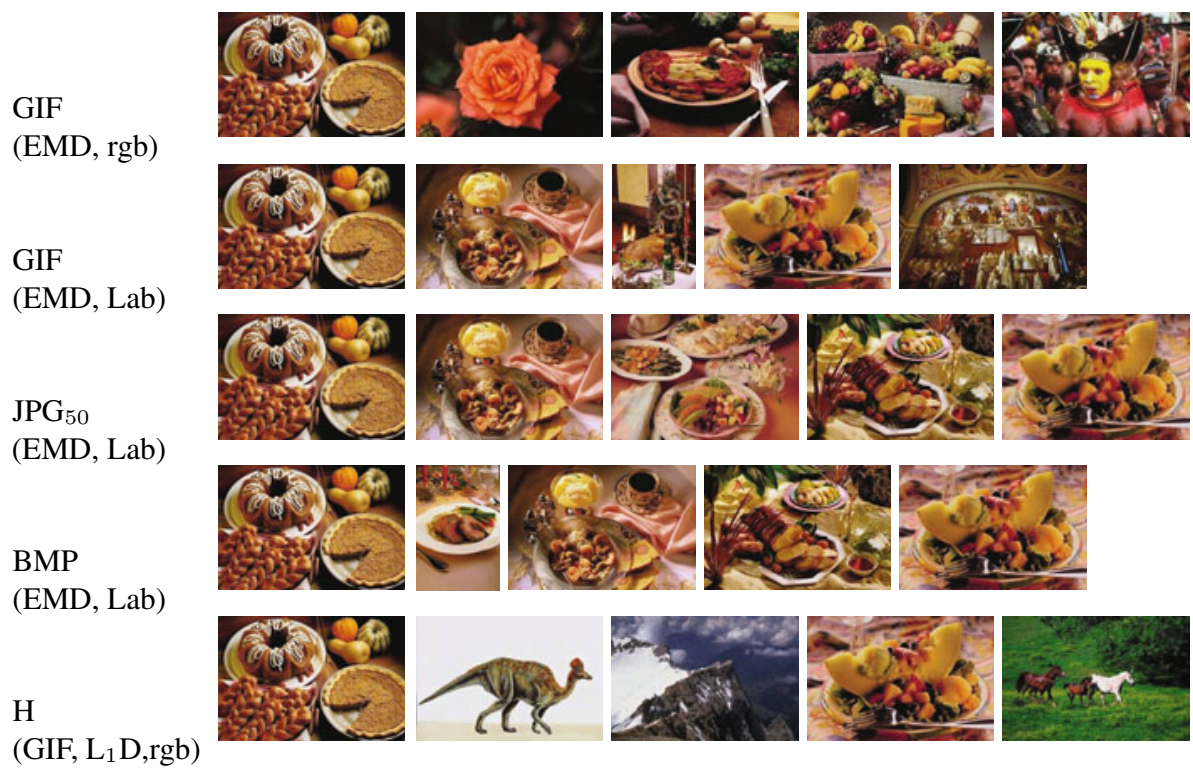

Fig. 5. Illustration of the retrieval results evaluated for images in GIF and JPG formats taken from the database of Wang using the rgb and Lab color space 
(a)
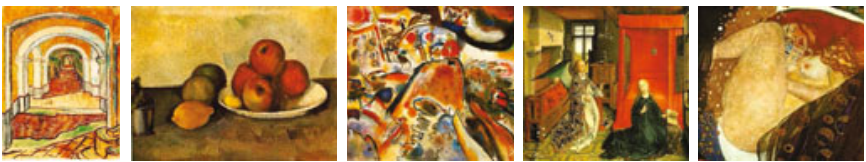

(b)
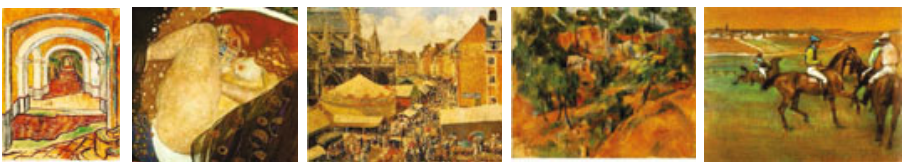

(c)
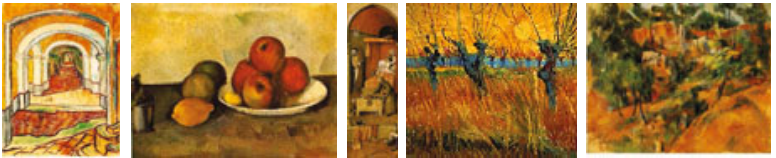

(d)
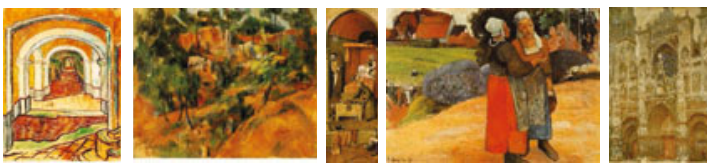

(e)
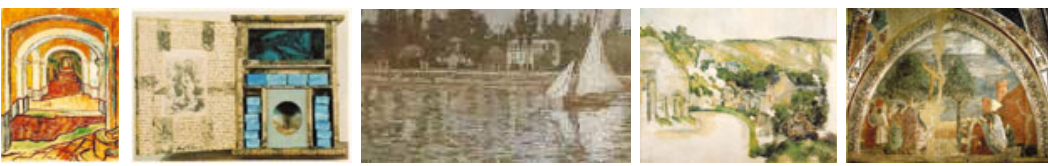

(a)
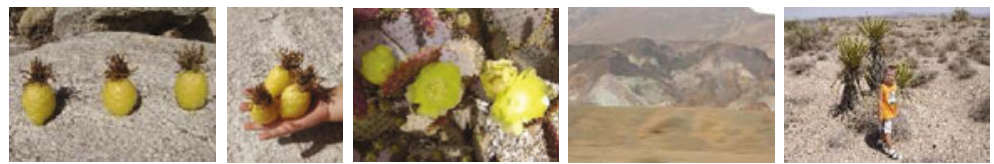

(b)
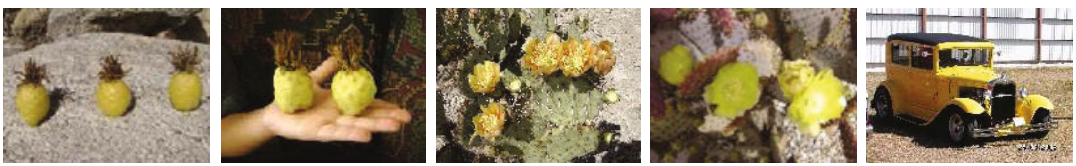

(c)
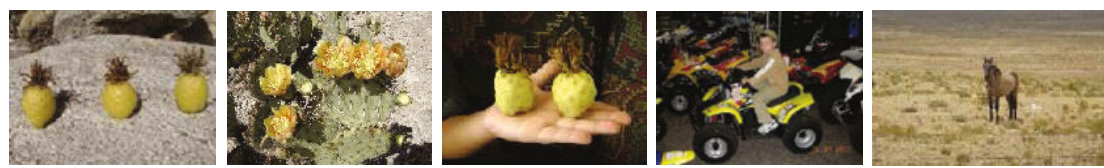

(d)
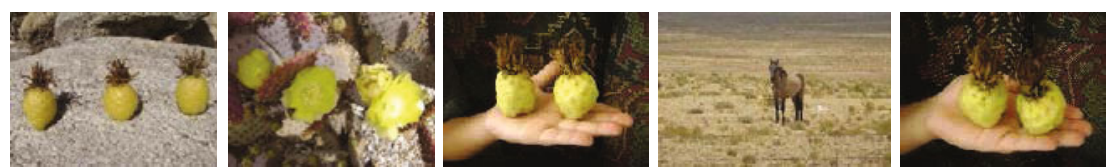

(e)
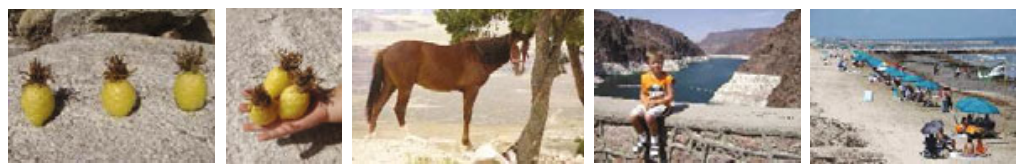

Fig. 6. Comparison of retrieval results using the Webmuseum and our database obtained for various retrieval parameters: GMM for ab histogram derived from CIE Lab with no additional weighting, using EMD as similarity measure (a), $k_{1,2}=1, h=0.3$ (b), $k_{1,2}=0.3, h=1$ (c), $k_{1,2}=2, h=0.3(\mathrm{~d})$, direct rg histogram comparison using $\mathrm{L}_{1} \mathrm{D}$ metric (e) 
(a)
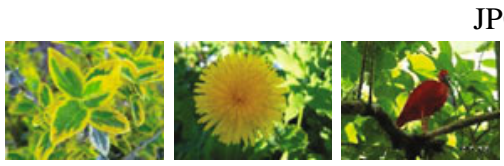

$\mathrm{JPG}_{15}$

(b)
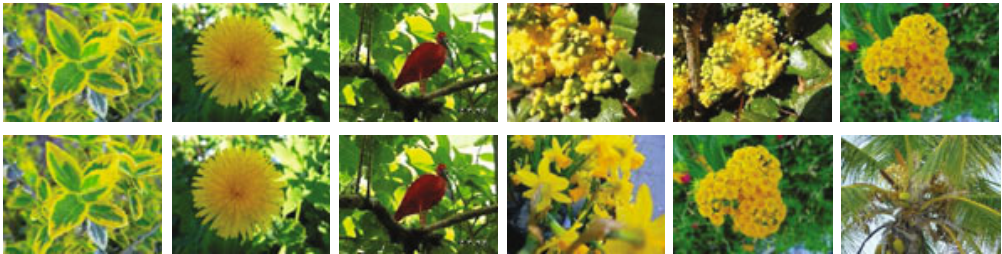

(c)
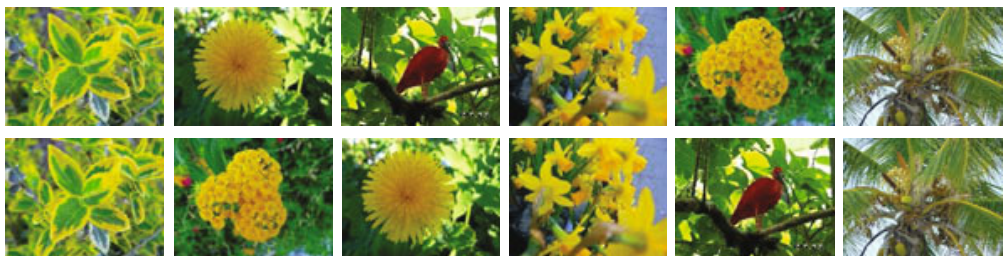

(d)
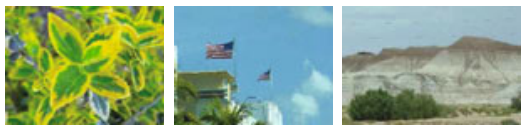

A.

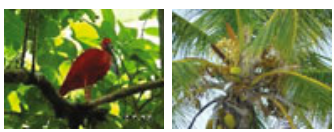

(e)
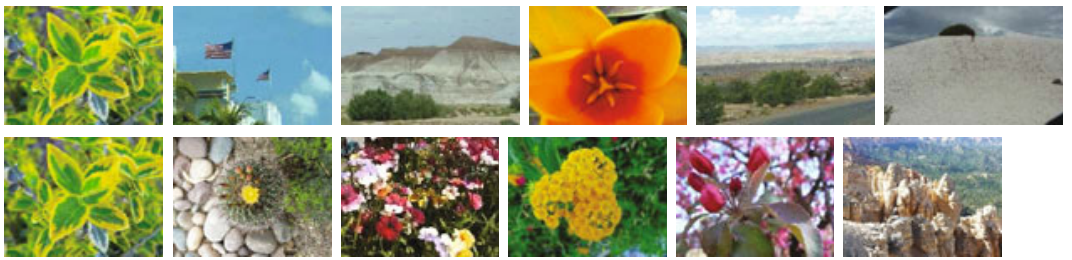

(f)
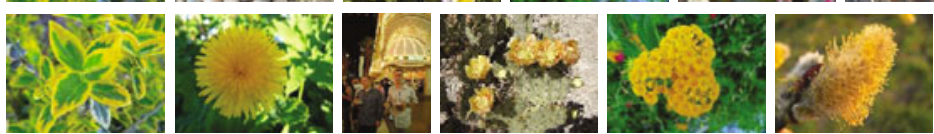

GIF

(a)
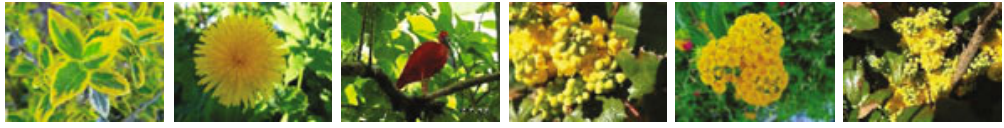

(b)
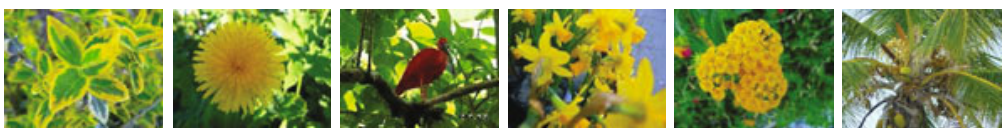

(c)
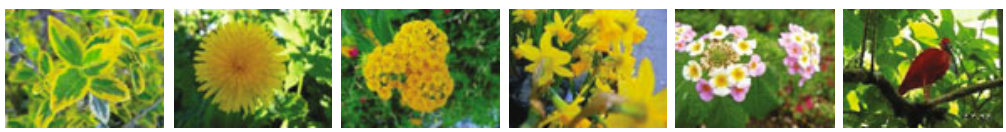

(d)
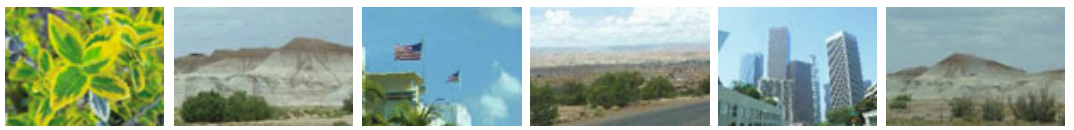

(e)
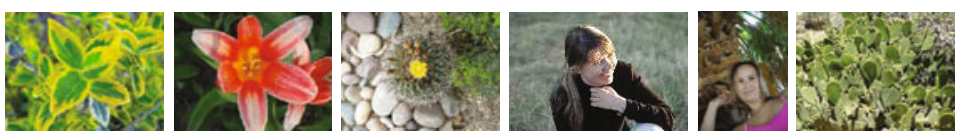

(f)
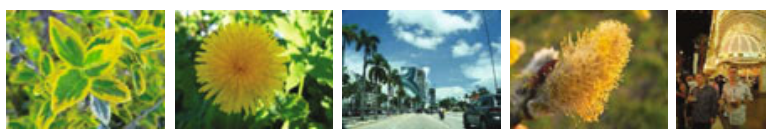

Fig. 7. The comparison of the retrieval results performed on our database obtained for various retrieval methods: GMM with no additional weighting applied for the images represented in the CIE Lab color space (a) CEDD [29] (b) FCTH [30] (c), MPEG-7 SCD [31] (d), Tamura histogram [32] (e), Haar wavelet (f). The results were evaluated for images transformed to $\mathrm{JPG}_{25}$, $\mathrm{JPG}_{15}$ and GIF. 


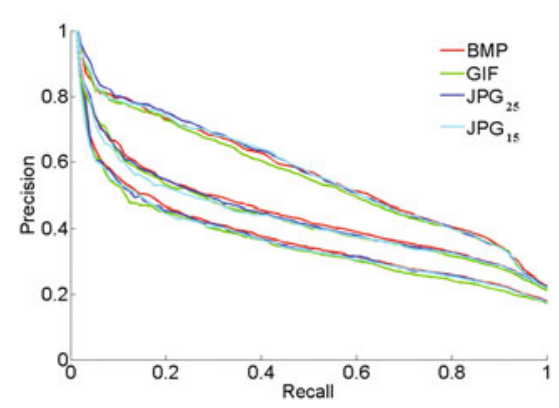

(a)

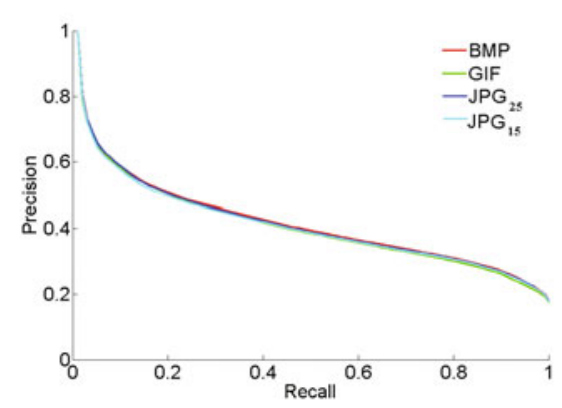

(b)

Fig. 8. Summary of the retrieval efficiency of the proposed method using the CIE Lab color space, evaluated for the database of Wang [8]. Plot (a) illustrates the retrieval power evaluated for three exemplary sets of 31 randomly chosen images. Plot (b) shows the retrieval results evaluated for the entire database of Wang.

\section{Evaluation of the Proposed Method Efficiency}

In order to test the proposed methodology we compared the retrieval results with those proposed by two well known, freely accessible image retrieval applications: img (Rummager) [28] and imgSeek, (www.imgseek.net). The first system comprises several methods among which the following were chosen: 60 bin histogram (CEDD) [29], 192 bin histogram (FCTH) [30], MPEG-7 [31] and Tamura [32] 16 bin directional histogram.

For the evaluation of results obtained using the GMM based technique we used the EMD method and the $\mathrm{L}_{1}$ metric for comparisons of the distance between the histograms. As can be seen in Fig. 7] only the CEDD [31] and FCTH methods yield similar results. The candidate images retrieved by the more sophisticated techniques (Fig. $7 \mathrm{~d}$-f) present distinctively different color palettes than that of the query and therefore they seem to be unsuitable for the purpose of the retrieval of lossy compressed images.

It is worth underlining that although the proposed solution outperforms, when lossy compressed images are analyzed, the other approaches discussed above, it is crucial to prove that it also yields valuable and comparable results for images of various levels of information loss. Thus, Fig. 8 depicts the robustness of the proposed method to the distortions introduced during compression process through Recall and Precision plots using the database of Wang. Independently on the applied compression scheme, the retrieval power is comparable with that when original images were subjected to retrieval process. In these experiments the criterion of the successful retrieval was the membership to the same thematic group as the query. Therefore, the main aim of the presented plots is to show that proposed technique can produce meaningful results regardlessly to the rate of information loss associated with various compression methods.

\section{Summary}

Many retrieval system rely on the color composition of the analyzed images. Although, this approach seems to be generally correct and effective, one must be aware of the 
problem of accurately managing the vast amount of visual information. The methods operating on chromaticity histograms could be severely disabled as color palettes of lossy compressed images can differ, providing misleading conclusions. As shown in this paper, such a comparison produces incorrect results when the retrieval process is evaluated not on the original images but on their compressed versions. Thus, there is an urgent need for the evaluation of techniques overcoming that undesirable phenomenon. Such a method is described in this paper.

The main contribution of this work is the adaptation of the concept of the Gaussian Mixture Models and the application of the Bilateral Filtering for the purposes of the distorted chromaticity histogram approximation. The proposed scheme enables the retrieval system not only to take into account the overall image color palette but also to consider the the color dispersion understood as spatial arrangement of image colors .

The satisfactory retrieval results were achieved independently on the applied compression scheme. Therefore, the presented results proved the hypothesis that the loss of color information caused by lossy coding can be efficiently counteracted providing successful retrieval results.

Acknowledgments. This work has been supported by the Polish Ministry of Science and Higher Education under R\&D grant no. N N516 374736 from the Science Budget 2009-2011.

\section{References}

1. Datta, R., Joshi, D., Li, J., Wang, J.Z.: Image Retrieval: Ideas, Influences, and Trends of the New Age. ACM Computing Surveys 40(2), 1-60 (2008)

2. Zhou, X.S., Rui, Y., Huang, T.S.: Exploration of Visual Data. Kluwer, Dordrecht (2003)

3. Pass, G., Zabih, R.: Comparing images using joint histograms. Journal of Multimedia Systems 7(3), 234-240 (1999)

4. Ciocca, G., Schettini, L., Cinque, L.: Image Indexing and Retrieval Using Spatial Chromatic Histograms and Signatures. In: Proc. of CGIV, pp. 255-258 (2002)

5. Lambert, P., Harvey, N., Grecu, H.: Image Retrieval Using Spatial Chromatic Histograms. In: Proc. of CGIV, pp. 343-347 (2004)

6. Hartut, T., Gousseau, Y., Schmitt, F.: Adaptive Image Retrieval Based on the Spatial Organization of Colors. Computer Vision and Image Understanding 112, 101-113 (2008)

7. Heidemann, G.: Combining Spatial and Colour Information For Content Based Image Retrieval. Computer Vision and Image Understanding 94, 234-270 (2004)

8. Wang, J.Z., Li, J., Wiederhold, G.: SIMPLIcity: Semantics-Sensitive Integrated Matching for Picture Libraries. IEEE Trans. Patt. Anal. Mach. Intel. 9, 947-963 (2001)

9. Rugna, J.D., Konik, H.: Color Coarse Segmentation and Regions Selection for Similar Images Retrieval. In: Proc. of CGIV, pp. 241-244 (2002)

10. Dvir, G., Greenspan, H., Rubner, Y.: Context-Based Image Modelling. In: Proc. of ICPR, pp. 162-165 (2002)

11. Jing, F., Li, M., Zhang, H.J.: An Effective Region-Based Image Retrieval Framework. IEEE Trans. on Image Processing 13(5), 699-709 (2004)

12. Berretti, A., Del Bimbo, E.: Weighted Walktroughs Between Extended Entities for Retrieval by Spatial Arrangement. IEEE Trans. on Multimedia 3(1), 52-70 (2002) 
13. Dempster, A., Laird, N., Rubin, D.: Maximum Likelihood from incomplete data. J. Royal Stat. Soc. 39B, 1-38 (1977)

14. McLachlan, G., Peel, D.: Finite Mixtures Models. John Wiley \& Sons, Chichester (2000)

15. Kuo, W.-J., Chang, R.-F.: Approximating the Statistical Distribution of Color Histogram for Content-based Image Retrieval. In: Proc. of ICASP, vol. 4, pp. 2007-2010 (2000)

16. Jeong, S.: Image Retrieval Using Color Histograms Generated by Gauss Mixture Vector Quantization. Comp. Vis. Image Understanding 94(1-3), 44-66 (2004)

17. Luszczkiewicz, M., Smolka, B.: Gaussian Mixture Model Based Retrieval Technique for Lossy Compressed Color Images. In: Kamel, M.S., Campilho, A. (eds.) ICIAR 2007. LNCS, vol. 4633, pp. 662-673. Springer, Heidelberg (2007)

18. Luszczkiewicz, M., Smolka, B.: A Robust Indexing and Retrieval Method for Lossy Compressed Color Images. In: Proc. of IEEE International Symposium on Image and Signal, Processing and Analysis, pp. 304-309 (2007)

19. Luszczkiewicz, M., Smolka, B.: Spatial Color Distribution Based Indexing and Retrieval Scheme. Advances in Soft Computing 59, 419-427 (2009)

20. Luszczkiewicz, M., Smolka, B.: Application of Bilateral Filtering and Gaussian Mixture Modeling for the Retrieval of Paintings. In: Proc. of ICIP, pp. 77-80 (2009)

21. Smolka, B., Szczepanski, M., Lukac, R., Venetsanoloulos, A.N.: Robust Color Image Retrieval for the World Wide Web. In: Proc. of ICASSP, pp. 461-464 (2004)

22. Elad, M.: On the Origin of the Bilateral Filter and Ways to Improve It. IEEE Trans. on Image Processing 11(10), 1141-1151 (2002)

23. Paris, S., Durand, F.: A Fast Approximation of the Bilateral Filter Using a Signal Processing Approach. Int. J. Comput. Vision 39B, 1-38 (2007)

24. Alata, O., Quintard, L.: Is There a Best Color Space for Color Image Characteriation or Representation Based on Multivariate Gaussian Mixture Model? Comp. Vis. Image Understanding 113, 867-877 (2009)

25. Kuehni, R.G.: Color Space and Its Divisions. John Wiley \& Sons, Chichester (2003)

26. Bilmes, J.: A Gentle Tutorial on the EM Algorithm and its Application to Parameter Estimation for Gaussian Mixture and Hidden Markov Models. University of Berkeley, ICSI-TR-97021 (1997)

27. Rubner, Y., Tomasi, C., Guibas, L.J.: A Metric for Distributions with Applications to Image Databases. In: Proc. of ICCV, pp. 59-66 (1998)

28. Chatzichristofis, S.A., Boutalis, Y.S., Lux M.: IMG(RUMMAGER): An Interactive Content Based Image Retrieval System. In: Proc. of the 2nd International Workshop on Similarity Search and Applications (SISAP), pp. 151-153 (2009)

29. Chatzichristofis, S.A., Boutalis, Y.S., Lux, M.: EDD: Color and Edge Directivity Descriptor, a Compact Descriptor for Image Indexing and Retrieval. In: Gasteratos, A., Vincze, M., Tsotsos, J.K. (eds.) ICVS 2008. LNCS, vol. 5008, pp. 312-322. Springer, Heidelberg (2008)

30. Chatzichristofis, S.A., Boutalis, Y.S., Lux, M.: FCTH: Fuzzy Color and Texture Histogram - a Low Level Feature for Accurate Image Retrieval. In: Proc. of the 9th International Workshop on Image Analysis for Multimedia Interactive Services (WIAMIS), pp. 191-196 (2008)

31. Manjunath, B.S., Ohm, J.R., Vasudevan, V., Yamada, A.: Color and Texture Descriptors. IEEE Trans. Cir. Sys. Video Technology 11, 703-715 (1998)

32. Tamura, S.M.H., Yamawaki, T.: Textural Features Corresponding to Visual Perception. IEEE Trans. Syst. Man Cybern. 8(6), 460-472 (1978) 